\title{
Arteria Vertebral Izquierda Aberrante
}

\author{
Aberrant Left Vertebral Artery
}

*Oscar Inzunza H. \& **́lvaro Burdiles

\begin{abstract}
INZUNZA, H. O. \& BURDILES, A. Arteria vertebral izquierda aberrante. Int. J. Morphol., 28(1):209-212, 2010.
\end{abstract}
RESUMEN: Se presenta una variación anatómica de la arteria vertebral izquierda, detectada en un cadáver utilizado para docencia en el Departamento de Anatomía de la Escuela de Medicina de la Pontificia Universidad Católica de Chile. En el mediastino superior del cadáver mencionado, se pudo apreciar que el arco aórtico da origen, de derecha a izquierda, a las siguientes ramas: arteria braquiocefálica, arteria carótida común izquierda, arteria vertebral izquierda aberrante y, finalmente, la arteria subclavia izquierda. La arteria vertebral izquierda aberrante se origina en la parte más alta del arco aórtico, presentando en este punto un diámetro de 4 mm; luego asciende a la izquierda de la carótida común ipsilateral y por sobre la cara anterolateral de la columna vertebral, tomando contacto con ramos cardíacos del tronco simpático cervical. Luego de un trayecto de $95 \mathrm{~mm}$, ocupando el mediastino superior y la raíz del cuello,

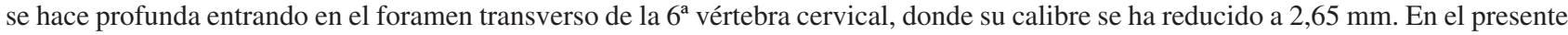
trabajo, se discute la evolución que presentan las arterias intersegmentarias dorsales cervicales en la antímera izquierda que permite explicar esta variación anatómica.

PALABRAS CLAVE: Variación anatómica; Arteria vertebral aberrante.

\section{INTRODUCCIÓN}

Si bien en ambas antímeras la arteria vertebral es clásicamente descrita como la primera rama de la arteria subclavia, la literatura especializada reporta múltiples variaciones en el origen de ella. Así, la arteria vertebral puede surgir del arco aórtico, desde la arteria carótida común o sus ramas, o desde alguna rama de la arteria subclavia, como el tronco tirocervical. También puede originarse a partir de dos ramas que confluyen, una desde el arco aórtico y otra desde la arteria subclavia (Lemke et al., 1999). Si bien estas variaciones afectan mayoritariamente a la arteria vertebral izquierda, algunos reportes describen el origen desde el arco aórtico para ambas arterias vertebrales (Lemke et al.; Albayram et al., 2002; Goray et al., 2005).

Aún cuando en la mayoría de los casos estas variaciones son asintomáticas y su hallazgo incidental, algunos autores han sugerido que el origen anómalo de las arterias vertebrales, sumado a un trayecto vascular más extenso, pueden provocar perturbaciones hemodinámicas que predisponen al portador de ellas a enfermedades cerebro vasculares (Lemke et al.; Goray et al.).
En este caso presentamos una variación anatómica del arco aórtico, en un cadáver humano de sexo masculino, el cual presenta cuatro ramas, donde la arteria vertebral izquierda se origina directamente como la tercera rama del arco aórtico. En su trayecto en el mediastino superior, la arteria se dispone entre la arteria carótida común y la subclavia del lado izquierdo.

El hallazgo de estas variaciones anatómicas en cadáveres utilizados con fines docentes en el Departamento de Anatomía de la Escuela de Medicina de la Pontificia Universidad Católica de Chile es especialmente atractivo para los alumnos y para los docentes, motivándonos a darlas a conocer en múltiples oportunidades (Inzunza et al., 1996a; 1996b; 2006). Esto permite resaltar lo formativo de la disección anatómica; método no superado para aprender, realmente, anatomía humana (Bravo \& Inzunza, 1995). Además, estas variaciones permiten a los alumnos aplicar los conocimientos de embriología para explicar su génesis (Inzunza et al., 2006).

\footnotetext{
* Profesor Titular, Departamento de Anatomia, Escuela de Medicina, Pontifícia Universidad Católica de Chile.

${ }^{* *}$ Profesor Asistente, Departamento de Radiología, Escuela de Medicina, Pontifícia Universidad Católica de Chile.
} 


\section{MATERIAL Y MÉTODO}

En un cadáver de sexo masculino, de 54 años de edad, cuya causa de muerte fue "complicaciones de una tuberculosis avanzada que terminó con paro cardíaco", utilizado para fines docentes en nuestro Departamento de Anatomía, se realizó una disección de mediastino superior. Se identificaron las ramas del arco aórtico y se siguió el trayecto de la arteria vertebral izquierda aberrante desde su origen hasta su entrada en el foramen transverso de la $6^{a}$ vértebra cervical. Luego, se procedió a inyectar silicona en la arteria, para poder medir con pie de metro su calibre tanto en su origen como al final de su trayecto. Asimismo, se midió su longitud total en su recorrido por el mediastino superior y por la raíz del cuello.

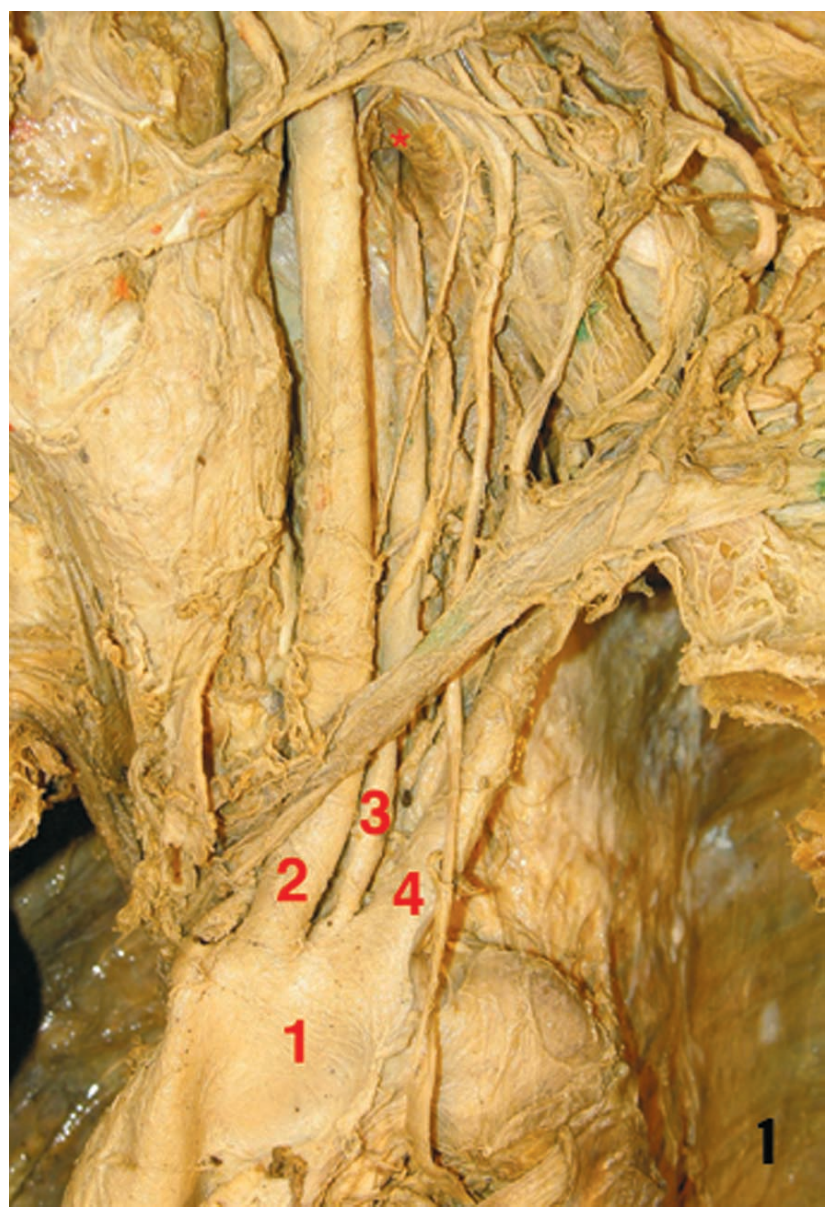

Fig. 1. Fotografía del arco aórtico (1) que da origen hacia la antímera izquierda a las ramas: arteria carótida común (2), arteria vertebral izquierda (3) y arteria subclavia izquierda (4). La arteria vertebral izquierda después de un largo trayecto aberrante ingresa en el foramen del proceso transverso de la sexta vértebra cervical $(*)$.

\section{RESULTADOS}

$\mathrm{La}$ arteria vertebral izquierda aberrante se origina en la parte más alta del arco aórtico, presentando en este punto un diámetro de $4 \mathrm{~mm}$; luego asciende situada a la izquierda de la carótida común y a la derecha de la subclavia ipsilaterales, tomando un curso oblicuo hacia cefálico, dorsal y lateral. Transcurre por sobre la cara anterolateral de la columna vertebral, tomando relación con ramos cardíacos del tronco simpático cervical (Fig. 1).

El vaso aberrante tiene una longitud total de $95 \mathrm{~mm}$, medidos entre su origen en la aorta hasta su penetración a través del foramen transverso de la $6^{\mathrm{a}}$ vértebra cervical. Es notable que, a medida que asciende, la arteria vertebral izquierda aberrante presenta una disminución progresiva de su calibre, comenzando con un diámetro de $4 \mathrm{~mm}$ en el arco aórtico, para llegar a un diámetro de 2,65 $\mathrm{mm}$ al final de su trayecto antes de ingresar en el foramen transverso.

En la otra antímera del cadáver, la arteria vertebral derecha nace desde la primera porción de la arteria subclavia, en un tronco común con la arteria intercostal superior, presentado en su origen un diámetro de $1,85 \mathrm{~mm}$.

\section{DISCUSIÓN}

Las variaciones del arco aórtico ofrecen una gran variabilidad de presentación, siendo la mayoría de ellas detectadas incidentalmente. Así, en un temprano reporte del siglo pasado, publicado en el primer número de los Archivos Chilenos de Morfología (1933), el Dr. Nicolás Muñoz Hidalgo grafica elegantemente al menos siete tipos de variaciones del arco aórtico, observadas en una serie de 300 disecciones de cadáveres femeninos, en las cuales incluye tres variedades que afectan a las arterias vertebrales.

Si bien el hallazgo de variaciones en el origen de las arterias vertebrales, tanto en angiografías y tomografías (Figs. 2 y 3) como en disecciones cadavéricas, es fortuito y es generalmente asintomática, su diagnóstico es relevante especialmente en aquellos pacientes en que se está considerando realizar una cirugía vascular en la región cervical, del arco aórtico, intervenciones endovasculares o una toracoscopía; como también en los pacientes portadores de malformaciones arteriovenosas o aneurismas cerebrales (Lemke et al.; Goray et al.).

El origen anómalo de la arteria vertebral izquierda desde el arco aórtico es una condición poco común; estudios 


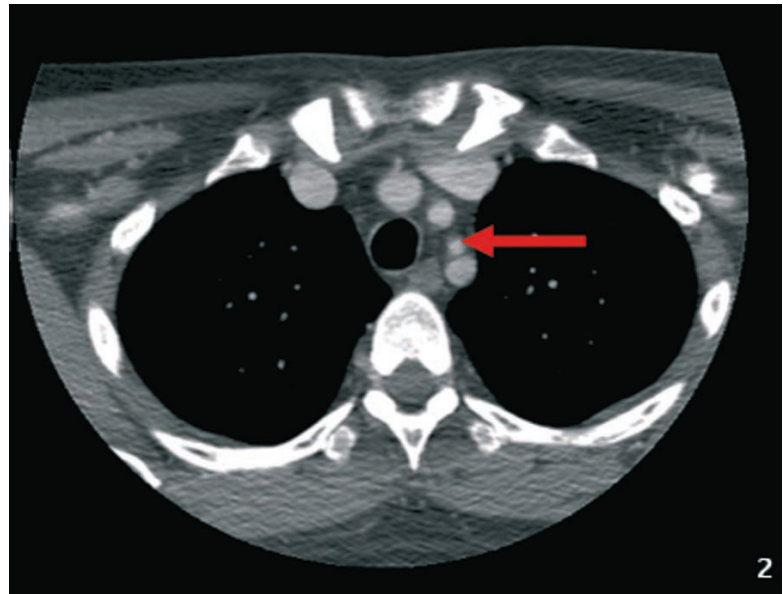

Fig. 2. Tomografía axial computarizada del mediastino superior de un paciente de sexo masculino, de 32 años, que presenta el trayecto aberrante de la arteria vertebral izquierda. La arteria (flecha roja) se dispone entre la carótida común y la arteria subclavia de la antímera izquierda.

foráneos, realizados en autopsias, la reportan entre el 2,4 y el 5,8\% de los casos (Newton \& Mani, 1974; Lemke et al.; Komiyama et al., 2001). Por otro lado, estudios radiográficos señalan que "en el 6-8\% la arteria vertebral izquierda nace directamente del arco aórtico, proximal a la arteria subclavia izquierda" (Rumack et al., 1999). En nuestro medio, el porcentaje de aparición de esta anomalía tiende a ser mayor. Así, como lo citamos anteriormente, en un estudio del siglo pasado (Muñoz), este hallazgo se detectó en el 8\% de 300 disecciones de cadáveres femeninos. Por su parte, del Sol \& Olave (1998) presentan cifras estadísticas bastante más elevadas, observando esta variación anatómica en 3 de 12 cadáveres humanos de sexo masculino (25\%) utilizados para docencia en la Facultad de Medicina de la Universidad de la Frontera, en Temuco. Los datos numéricos de estos autores sugieren una posible causa étnica en la aparición de esta anomalía.

Komiyama et al. han reportado una elevada incidencia de lesiones arteriales disecantes asociadas con el origen anómalo de la arteria vertebral izquierda desde el arco aórtico; dato preocupante en nuestro medio por las razones expuestas en el párrafo precedente. Estos autores argumentan que en estos casos la arteria podría recibir directamente la fuerza pulsátil del corazón, mientras que la arteria vertebral originada desde la arteria subclavia recibiría un flujo más atenuado. Asimismo, el extenso trayecto de la arteria aberrante podría afectar la resistencia hemodinámica del vaso, provocando una fatiga en la porción distal de la arteria.

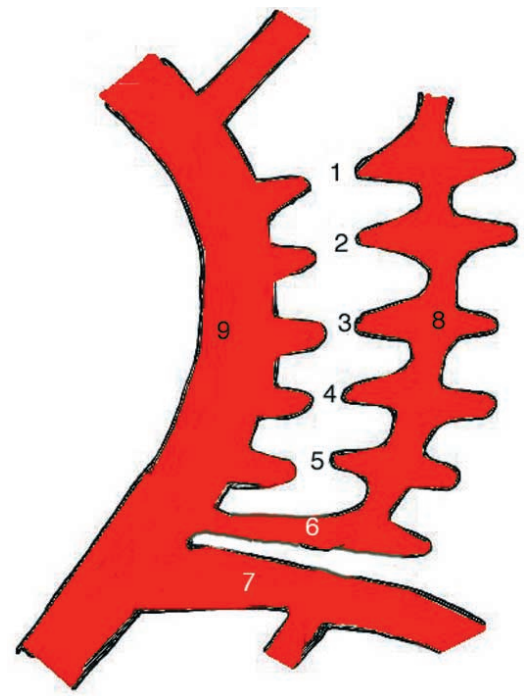

Fig. 4.- Esquema modificado de Moore y Persaud que ilustra la probable explicación del origen anómalo de la arteria vertebral izquierda. 1 a $5=$ arterias intersegmentales cervicales primera a quinta. $6=$ sexta arteria intersegmental cervical que retiene su conexión con la aorta dorsal izquierda y con la anastomosis longitudinal. $7=$ séptima arteria intersegmental que da origen a la subclavia. $8=$ Anastomosis longitudinal que da origen a la arteria vertebral. $9=$ Aorta dorsal izquierda.

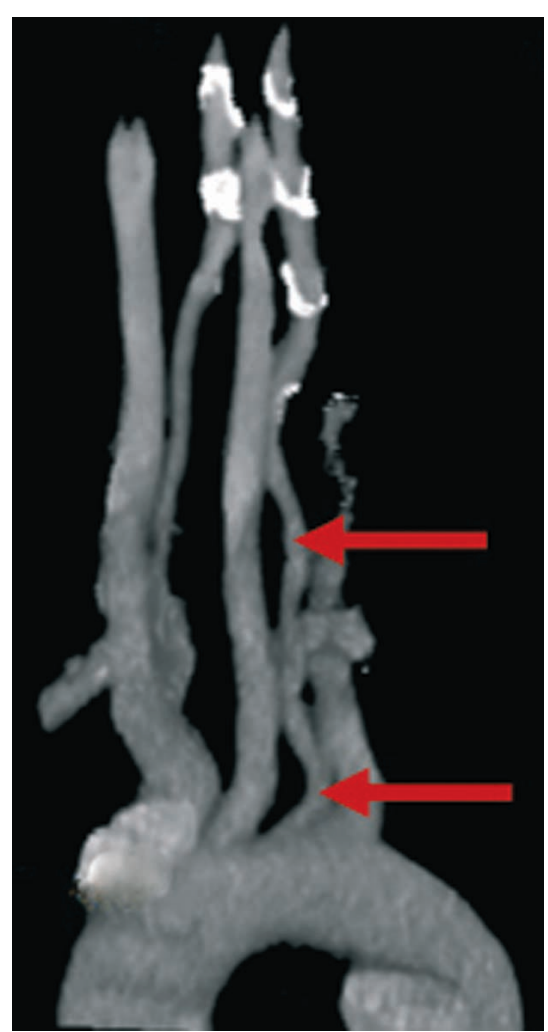

Fig. 3. Imagen reformateada 3D del caso mostrado en la Figura 2, que muestra el origen en el arco aórtico de la arteria vertebral izquierda y su largo trayecto en el mediastino superior y en la raíz del cuello (flechas rojas).

Embriológicamente, las arterias vertebrales se forman, en cada antímera, a partir del desarrollo de las anastomosis longitudinales que conectan a las arterias intersegmentales cervicales, ramas de las aortas dorsales. Las arterias intersegmentales sufren regresión con excepción de la séptima intersegmental, la cual formará la porción proximal de la arteria subclavia, reteniendo el extremo caudal de la anastomosis longitudinal; punto de origen, en la subclavia, de la arteria vertebral en el adulto (Newton \& Mani; Larsen, 1998). El origen aberrante de la arteria vertebral izquierda desde el arco aórtico se explica por una persistencia de la sexta arteria intersegmental (Fig. 4), la cual retiene la conexión con la anastomosis longitudinal (Albayram et al.). 
INZUNZA, H. O. \& BURDILES, A. Aberrant left vertebral artery. Int. J. Morphol., 28(1):209-212, 2010.

SUMMARY: An anatomical variation of the left vertebral artery, detected in a cadaver used for teaching purposes in the Department of Anatomy, School of Medicine, Catholic University of Chile is presented. In this case, the aortic arch gives rise, from right to left, to the following branches: the brachiocephalic artery, the left common carotid artery, the aberrant vertebral left artery and, finally, the left subclavian artery. The aberrant left vertebral artery originates from the top of the aortic arch, presenting here a diameter of $4 \mathrm{~mm}$, then ascends to the left of the common carotid ipsilateral, over the anterolateral aspect of the spine, making contact with cardiac branches of the cervical sympathetic trunk. After ascending $95 \mathrm{~mm}$, occupying the upper mediastinum and the root of the neck, the artery enters the transverse foramen of the 6 th cervical vertebra, where its diameter was reduced to $2.65 \mathrm{~mm}$. In this paper we discuss the development of the cervical dorsal intersegmental arteries in the left antimera, process that could explain this anatomical variation.

KEY WORDS: Anatomical variation; Aberrant vertebral artery.

\section{REFERENCIAS BIBLIOGRÁFICAS}

Albayram, S.; Gailloud, P. \& Wasserman, B. Bilateral arch origin of the vertebral artery. Am. J. Neuroradiol., 23:455-8, 2002.

Bravo, H. \& Inzunza, O. Evaluación de algunos programas computacionales en la enseñanza de Anatomía y Neuroanatomía de la Facultad de Medicina de la Pontificia Universidad Católica de Chile. Rev. Chil. Anat., 13(1):79-86, 1995.

Del Sol, M. \& Olave, E. Origen aórtico de la arteria vertebral en el hombre. Rev. Chil. Cienc. Méd. Biol., 8(1):313, 1998.

Goray, V. B.; Joshi, A.; Garg, A.; Merchant, S.; Yadav, B. \& Maheshwari P. Aortic arch variation: a unique case with anomaluos origin of both vertebral arteries as additional branches of the aortic arch distal to left subclavian artery. Am. J. Neuroradiol., 26:93-5, 2005.

Inzunza, O.; Prado, M. \& Valenzuela A. Variación del origen de la arteria coronaria derecha. Rev. Chil. Cienc. Med. Biol., 6(1):11-4 1996a.

Inzunza, O.; Valenzuela, A.; \& Prado, M. Anastomosis anómala entre las arterias hepática y mesentérica superior. Rev. Chil. Cienc. Biol., 6(2):65-8, 1996 b.

Inzunza, O.; Ortega, W.; Berrocal, C. \& Vargas, A. Variación anatómica de la valva atrioventricular derecha. Int. J. Morphol., 24(1):49-52, 2006.

Larsen, W. J. Essentials of Human Embryology. New York, Churchill Livingstone, 1998. pp. 146-7.

Lemke, A. J.; Benndorf, G.; Liebig, T. \& Felix, R. Anomalous
Origin of the Right Vertebral Artery: review of the literature and case report of the right vertebral artery origin distal to the left subclavian artery. Am. J. Neuroradiol., 20:1318-21, 1999.

Komiyama, M.; Morikawa, T.; Nakajima, H.; Nishikawa, M. \& Yasui, T. High incidence of arterial dissection associated with left vertebral artery of aortic origin. Neurol. Med. Chir., (Tokyo). 41(1):8-12, 2001.

Muñoz, N. Variedades de cayado aórtico en la mujer. Arch. Chil. Morfol., 1(1):27-37, 1933.

Newton, T. H. \& Mani, L. R. The Vertebral Artery. In: Newton, T. H. \& Potts, D. eds. Radiology of the Skull and Brain. St. Louis, Mosby, 1974. pp. 1659-72.

Rumack, C.; Wilson, S. \& Charboneau, J. Diagnóstico por Ecografía. 2a Ed. Editorial Marbán. 1999. p. 911.
Dirección para correspondencia:
Prof. Dr. Oscar Inzunza
Departamento de Anatomía Normal
Escuela de Medicina,
Pontificia Universidad Católica de Chile
CHILE

Teléfono 56-2-354 3076

Email: oinzunza@med.puc.cl

Recibido : 23-11-2009

Aceptado: 04-01-2010 